\title{
A NOTE ON PARTIAL VERTICAL INTEGRATION
}

\author{
George HENDRIKSE \\ Tilburg University, 5000 LE Tilburg, The Netherlands

\section{Hans PETERS} \\ University of Limburg, $6200 \mathrm{MD}$ Maastricht, The Netherlands
}

Received 25 August 1988

Accepted 26 October 1988

\begin{abstract}
A simple model is constructed to show how partial vertical integration may emerge as an equilibrium market structure in a world characterized by rationing, differences in the reservation prices of buyers, and in the risk attitudes of buyers and sellers. The buyers with the high reservation prices turn out to be vertically integrated.
\end{abstract}

\section{Introduction}

Empirical analysis [see, e.g., McDonald (1985)] suggests that partial vertical integration is a widespread phenomenon. However, there are hardly any theoretical explanations for this composition of industries. Both Arrow (1975) and Green (1986) explicitly allow for partial integration to occur. The former author takes the need for information to be the driving force behind vertical integration, whereas in the latter paper market advantages due to integration versus decreasing returns to an expansion of the scope of activities carried out by a single firm are the opposing forces. In both models, however, the industry equilibrium tends to either no integration at all, or complete vertical integration. Carlton (1979) obtained an intermediate outcome by having risk averse buyers secure their high probability demand. In the model of Quirmbach (1986), scale and/or substitution effects are responsible for partial vertical integration. If a firm switches from market exchange to vertical integration, then this will change the probability distribution of the spot market price. This externality is responsible for partial vertical integration in the model of Hendrikse (1988).

The present note is in the spirit of Green (1986). However, we will obtain partial vertical integration as an equilibrium market structure, mainly by taking the risk attitudes of the traders into considcration.

\section{The model}

The model under consideration consists of $m$ buyers and $m$ sellers. Each buyer always wants to buy one unit of a given fixed product. The reservation price of buyer $i$ for the product is $R_{i}$; we assume that $R_{1} \geqslant R_{2} \geqslant \cdots \geqslant R_{m}>0$. All sellers are assumed to be identical; each seller either has one unit of the product for sale, or noting at all, the probability of the first even being $q \in(0,1)$. 
Trade can take place either in a contract, or on the spot market. Following Green (1986), we assume that the price on the spot market is fixed. More precisely, we assume it to be equal to the lowest reservation price of the buyers present on the spot market; if the spot market is empty, then we define the spot market 'price' to be $R_{m}$.

We further assume that the market for the product is subject to stochastic fluctuations in exogenous excess demand, leading to rationing of the market. The mechanism by which the product will be rationed is taken to be identical to the one used by Green (1986). There will always be excess demand in our model; a fraction $q$ of the buyers will have their demands met exactly, the remaining buyers will receive nothing.

If a buyer and a seller vertically integrate, then their relationship is governed by a set of rules which form a binding agreement. The alternative to vertical integration is to be in the spot market. The rules governing vertical integration are:

(i) If, in a contract of a seller with a buyer $i$, the seller has a unit of the product of sale, then (s)he has to deliver this unit for a price $p_{i}$ to buyer $i$.

(ii) If, in the same contract, the seller does not have the product for sale, then both buyer and seller still receive their loss of surplus. These payments will be covered by insurance premiums $\pi^{\mathrm{b}}\left(R_{i}, p_{i}\right)$ and $\pi^{\mathrm{s}}\left(R_{i}, p_{i}\right)$, respectively.

Let $S$ denote the set of buyers and sellers on the spot market, and let $S^{\mathrm{c}}$ denote its complement, i.e., the set of buyers and sellers in contracts. ${ }^{1}$ Let $p(S)$ denote the price on the spot market. Let $\eta$ take the value 1 if the seller has a unit for sale, i.e., the buyer acquires the product, and let $\eta$ be 0 otherwise. We write the (pure) payoffs of buyer $i\left(b_{i}\right)$ and seller $i\left(s_{i}\right)$ as follows:

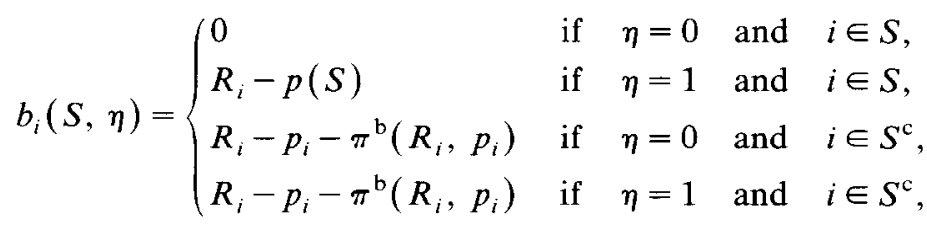

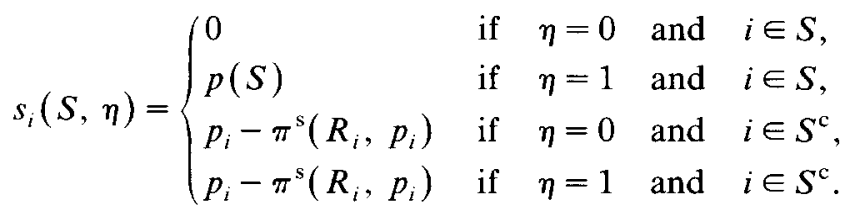

Let $v$ and $u$ denote the (von Neumann-Morgenstern) utility functions of sellers and buyers, respectively, normalized such that $u(0)=v(0)=0$. Our crucial assumption is that $v$ is (weakly) convex and $u$ is (weakly) concave, i.e., that buyers are (weakly) risk averse whereas sellers are (weakly) risk prone.

We call a market structure $S$ and equilibrium market structure ${ }^{2}$ if there exists a set of prices $\left\{p_{i}\right\}_{i \in S^{c}}$ such that:

$\forall i \in S^{\mathrm{c}}: u\left(R_{i}-p_{i}-\pi^{\mathrm{b}}\left(R_{i}, p_{i}\right)\right)>q u\left(R_{i}-p(S \cup\{i\})\right)$,

${ }^{1}$ Since in our model always exactly $m$ pairs of buyers and sellers will be formed, we (slightly mis)use the same letter to denote the buyer, the seller, or the pair, depending on the context.

2 The model could be formulated as a noncooperative game in which partial vertical integration emerges as a Nash equilibrium. However, this would require much more notation. 
$\forall i \in S^{\mathrm{c}}: v\left(p_{i}-\pi^{\mathrm{s}}\left(R_{i}, p_{i}\right)\right)=q v(p(S \cup\{i\}))$,

$\sum_{i \in S^{\mathrm{c}}} \pi^{\mathrm{b}}\left(R_{i}, p_{i}\right)+\pi^{\mathrm{s}}\left(R_{i}, p_{i}\right)=\sum_{i \in S^{\mathrm{c}}}(1-q) R_{i}$,

and such that for no $j \in S$ there is a price $p_{j}$ with

$u\left(R_{j}-p_{j}-\pi^{\mathrm{b}}\left(R_{j}, p_{j}\right)\right)>q u\left(R_{j}-p(S)\right)$,

$v\left(p_{j}-\pi^{\mathrm{s}}\left(R_{j}, p_{j}\right)\right)=q v(p(S))$,

$\sum_{i \in S^{\mathrm{c}} \cup\{j\}} \pi^{\mathrm{b}}\left(R_{i}, p_{i}\right)+\pi^{\mathrm{s}}\left(R_{i}, p_{i}\right)=\sum_{i \in S^{\mathrm{c}} \cup\{j\}}(1-q) R_{i}$.

In writing down the above conditions, we use the fact that $u$ and $v$ are von Neumann-Morgenstern utility functions, which assign expected utilities to risky events. Conditions (3) and (6) are insurance balance conditions. Conditions (1) and (2) keep buyers and sellers from breaking contracts and wander off to the spot market, conditions (4) and (5) prevent the reverse phenomenon. In (2) and (5) we use equality signs: this is a reasonable simplification in view of the fact that all sellers were supposed to be identical. The strict inequality signs in (1) and (4) reflect out assumption that, in case of indifference, a buyer chooses to be in the spot market.

In what follows, we will assume that the insurance premiums have the following simple forms:

$\pi^{\mathrm{s}}\left(R_{i}, p_{i}\right)=\pi p_{i}, \quad \pi^{\mathrm{b}}\left(R_{i}, p_{i}\right)=\pi\left(R_{i}-p_{i}\right) \quad$ for some $\quad 0<\pi<1$

That is, each party covers its own risk.

Proposition 1. Suppose the insurance premiums are given by (7). Then:

(a) If $\pi \neq 1-q$, then there exists a unique equilibrium market structure $S_{*}$, given by $S_{*}^{\mathrm{c}}=\emptyset$.

(b) Suppose $\pi=1-q$. Then there exists a unique equilibrium market structure $S_{*}$, which has the following form: there is an $0 \leqslant i_{S_{*}}<m$ with $i \in S_{*}$ if and only if $i>i_{S_{*}}$.

Proof. (a) follows immediately from (3) and (6). We now assume $\pi=1-q$. We first prove the following statement: If $S$ is an equilibrium market structure and $S^{c} \neq \emptyset$, then

$S=\left\{i: i>i_{s}\right\}$

where $i_{S}:=\max \left\{i: i \in S^{\mathrm{c}}\right\}$ and $\max \emptyset:=0$.

Let $S$ be as in the antecedent of (8). If $S=0$, then we are done. So we assume $S \neq 0$. Note that $p_{i_{s}}$ solves solves (2), i.e., $v\left(q p_{i_{s}}\right)=q v\left(p\left(S \cup\left\{i_{s}\right\}\right)\right)=q v\left(R_{m}\right)$. Here, the last equality follows from the definition of $i_{s}$. Also note that $i_{s}<m$; otherwise, since the convexity of $v$ implies $p_{i_{s}} \geqslant R_{m}$, we would have $p_{m} \geqslant R_{m}$, which implies that (1) would be violated. Now suppose there is a $j<i_{S}$ with $j \in S$. Define $p_{J}=p_{i_{s}}$. Then, since $p(S)=p\left(S \cup\left\{i_{s}\right\}\right)=R_{m}$, the equations in (5) as well as in (6) are satisfied for this $j$, so the equation in (4) cannot hold, i.e.:

$u\left(q\left(R_{j}-p_{j}\right)\right) \leqslant q u\left(R_{j}-R_{m}\right)$. 
By (1) we also have

$u\left(q\left(R_{i_{\mathrm{s}}}-p_{j}\right)\right)>q u\left(R_{i_{\mathrm{s}}}-R_{m}\right)$.

We set $a=R_{i_{s}}-p_{j}, b=R_{i_{s}}-R_{m} \geqslant a$. Then $R_{j}-p_{j}=a+\epsilon$ and $R_{j}-R_{m}=b+\epsilon$ for some $\epsilon \geqslant 0$. Rewriting (9) and (10) we obtain

$u(q(a+\epsilon)) \leqslant q u(b+\epsilon)$,

$u(q a)>q u(b)$.

From (11), (12), and the concavity of $u$, we derive $[q u(b+\epsilon)-q u(b)>u(q(a+\epsilon))-u(q a) \geqslant u(a+$ $q \epsilon)-u(a)=u(q(a+\epsilon)+(1-q) a)-u(a) \geqslant q u(a+\epsilon)-q u(a)$. $]$ hence $[u(b+\epsilon)-u(b)>u(a+\epsilon)$ $-u(a)$.] The concavity of $u$ then implies $b<a$, a contradiction. This completes the proof of (8).

Now let $S_{*}$ be an equilibrium market structure. First consider the case that $S_{*}^{\mathrm{c}}=\emptyset$, i.e., $i_{S_{*}}=0$. Suppose there was another equilibrium market structure $S$. Then $S^{\mathrm{c}} \neq \emptyset$. In particular, by (8), $1 \in S^{\mathrm{c}}$ and $p(S)=R_{m}=p\left(S_{*}\right)$. So there is a $p_{1}$ such that (1) and (2) hold for $1 \in S^{\mathrm{c}}$. But then, by taking $j=1$ and $p_{j}=p_{1}$, we have a violation of (4)-(6) applied for $S_{*}$. In the other case, where $S_{*}^{\mathrm{c}} \neq \emptyset$, we prove uniqueness of the equilibrium in an analogous way.

In order to show existence, we determine the (constant) eventual contract price $p_{\imath}$ by solving (2), that is $v\left(q p_{i}\right)=q v\left(R_{m}\right)$. Next, we let $i_{s}$ be the largest index $i$ for which (1) can be satisfied, so $i_{S}=\max \left\{i: u\left(q\left(R_{i}-p_{i}\right)\right)>q u\left(R_{i}-R_{m}\right)\right\}$ with $\max \emptyset=0$. Then an equilibrium market structure is $S=\left\{i: i>i_{s}\right\}$.

The following simple example shows that nontrivial partial vertical integration may occur. Let there be three sellers and three buyers with $R_{1}=5, R_{2}=3, R_{3}=1$. Let $u(t)=\sqrt{t}(t \geqslant 0)$ and $v(t)=t$, and let $q=1-\pi=\frac{8}{10}$. Then the unique equilibrium market structure is given by $S=\{3\}$. Further: $p(S)=R_{3}=1, p_{1}=p_{2}=1$. This example also shows that for proper partial vertical integration to occur, sellers may be risk neutral. If $v(t)=t^{2}$, then we obtain the same equilibrium structure with prices $p_{1}=p_{2}=\frac{5}{4}$.

\section{Conclusion}

A simple model was constructed to show how partial vertical integration may emerge as an equilibrium market structure in an environment characterized by rationing, differences in the reservation prices of buyers, and in the risk attitudes of buyers and sellers.

\section{References}

Arrow, K.J. 1975, Vertical integration and communication, Bell Journal of Economics 6, 173-183.

Carlton, D.W., 1979, Vertical integration in competitive markets under uncertainty, The journal of the Industrial Economics 27, 189-209.

Green, J.K., 1986, Vertical integration and assurance of markets, in: J.E. Stiglitz and G.F. Mathewson, eds., New developments in the analysis of market structure (MIT Press, Cambridge, MA).

Hendrikse, G.W.J., 1988, On the coexistence of spot and contract markets (University of Limburg, Maastricht).

McDonald, J.M., 1985, Market exchange or vertical integration: An empirical analysis, Review of Economics and Statistics 67.

Quirmbach, H.C., 1986, Vertical integration: Scale distortions, partial integration and the direction of price change, Quarterly Journal of Economics 102, 131-147. 